\title{
AN ANALYTICAL SOLUTION TO JACOBSEN ESTIMATOR FOR WINDOWED SIGNALS
}

\author{
Takahiro Murakami ${ }^{\dagger \ddagger}$ and Wenwu Wang $\ddagger$ \\ ${ }^{\dagger}$ Department of Electronics and Bioinformatics, Meiji University, Japan \\ ${ }^{\ddagger}$ Centre for Vision, Speech and Signal Processing (CVSSP), University of Surrey, UK
}

\begin{abstract}
Interpolated discrete Fourier transform (DFT) is a well-known method for frequency estimation of complex sinusoids. For signals without windowing (or with rectangular-windowing), this has been well investigated and a large number of estimators have been developed. However, very few algorithms have been developed for windowed signals so far. In this paper, we extend the well-known Jacobsen estimator to windowed signals. The extension is deduced from the fact that an arbitrary cosine-sum window functions are composed of complex sinusoids. Consequently, the Jacobsen estimator for windowed signals can be formulated as an algebraic equation with no approximation and thus an analytical solution to the estimator can be obtained. Simulation results show that our approach improves the performance in comparison with the conventional interpolated DFT algorithms for windowed signals.
\end{abstract}

Index Terms - Frequency estimation, interpolated DFT, Jacobsen estimator, window function, analytical solution

\section{INTRODUCTION}

Frequency estimation of complex sinusoids is one of the important topics in various fields using digital signal processing. Interpolated discrete Fourier transform (DFT) is a well-known approach for such a purpose [1-11]. In the interpolated DFT, several DFT coefficients at a local peak on an amplitude spectrum and its vicinity are exploited. Typically only two or three DFT coefficients are used for frequency estimation. Hence, low computational cost is an attractive feature of the interpolated DFT.

Because the interpolated DFT is based on the peak search of the amplitude spectrum, frequency estimation of multiple complex sinusoids is achieved one by one. In this case, however, DFT coefficients around the local peak are influenced by the sidelobes of other complex sinusoids [9]. In order to alleviate the interference with the sidelobes on the DFT spectrum, window functions are generally applied to the signals. Therefore, development of algorithms of the interpolated DFT for windowed signals is effective in frequency estimation of practical signals. For the signals without windowing (or with rectangular-windowing), the interpolated DFT has been investigated extensively and a large number of estimators have been proposed so far [1-8]. However, very few methods of the interpolated DFT have been developed for windowed signals to date [9-11]. This is because mathematical complexity is massively increased by windowing.

In this paper, we develop an estimator based on the interpolated DFT for windowed signals. Our estimator is an extended version of the Jacobsen estimator [4] that is a well-known estimator for signals without windowing. The proposed approach focuses on cosine-sum window functions that include the commonly used Hanning, Hamming, and Blackman windows. Exploiting the fact that the cosine- sum windows can be expressed by multiple complex sinusoids, we translate the Jacobsen estimator into an algebraic equation with no approximation and as a result, an estimate of the frequency is given by an analytical solution. Especially when the Hanning and Hamming windows are used, the estimator is translated into a cubic equation. It is known that the estimation error by the interpolated DFT comprises two kinds of artifacts, i.e., distortion caused by additive noise and an estimator bias derived from approximation of functions in an estimator $[6,12]$. The former distortion is dominant in the low signal-to-noise ratio (SNR) environments while the latter bias is a determining factor when the SNR is high. Because the proposed algorithm includes no approximation, the estimator bias is thoroughly removed and hence the estimation error becomes effectively small when the SNR is high. In addition, this estimator can be applied to various signals because arbitrary cosine-sum windows are allowed.

Related to our work are the Candan estimator [9] and the Liao estimator [10] which are extension of the Jacobsen estimator to windowed signals. The Candan estimator achieves the extension using the first degree Taylor polynomial of the DFT of the window function. Although the Candan estimator can be applied to any window functions in addition to the cosine-sum window functions, the performance deteriorates as the SNR increases due to the estimator bias caused by the truncation of the Taylor series expansion. The Liao estimator gives an analytical solution to the slightly altered Jacobsen estimator when the Hanning window is employed. Because the Liao estimator is derived with no approximation, the estimator bias doesn't occur and hence the performance is improved in the case of high SNR environments. However, only the Hanning window is acceptable in the Liao estimator. In contrast, our approach has advantages in these aspects over these conventional estimators.

\section{PRELIMINARIES}

Let $y(k)$ be an $N$-point discrete-time observation of a complex sinusoid modeled as

$$
y(k)=x(k)+n(k),(k=0,1, \ldots, N-1),
$$

where

$$
x(k)=a e^{j b} e^{j 2 \pi f k}
$$

is a complex sinusoid with unknown amplitude $a(a>0)$, an unknown initial phase $b(|b| \leq \pi)$, and an unknown normalized frequency $f(0<f<1), n(k)$ is an additive noise, $k$ indicates a discrete-time index, and $j$ is the imaginary unit. Then, the discrete Fourier transform (DFT) of $x(k)$ can be written by [13]

$$
X(m)=a e^{j b} e^{j \pi\left(f-\frac{m}{N}\right)(N-1)} \frac{\sin \left(\pi N\left(f-\frac{m}{N}\right)\right)}{\sin \left(\pi\left(f-\frac{m}{N}\right)\right)}
$$

Based on the maximum likelihood (ML) estimate [14], it is wellknown that maximization of the periodogram of $x(k)$ gives the true 
frequency $f$. Therefore, $f$ can be expressed as

$$
f=\frac{m_{0}+\delta}{N}
$$

where $m_{0}$ is the nearest integer to $(f N), \delta$ is the residual with $|\delta| \leq$ 0.5 , and $m_{0}$ can be given by the maximum points of $|X(m)|$ as

$$
m_{0}=\underset{m=0,1, \ldots, N-1}{\arg \max }|X(m)| .
$$

Estimation of $m_{0}$ and $\delta$ are often referred to as a coarse search and $a$ fine search, respectively. Using $m_{0}$ and an arbitrary integer $l$, Eq. (3) is rewritten by

$$
X\left(m_{0}+l\right)=\tilde{a} \frac{c_{l}+j s_{l}}{c_{l} \tilde{s}-s_{l} \tilde{c}}
$$

where $\tilde{a}=a e^{j b} e^{j \pi \frac{\delta}{N}(N-1)} \sin (\pi \delta), \tilde{s}=\sin \left(\pi \frac{\delta}{N}\right), \tilde{c}=\cos \left(\pi \frac{\delta}{N}\right)$, $s_{l}=\sin \left(\pi \frac{l}{N}\right)$, and $c_{l}=\cos \left(\pi \frac{l}{N}\right)$.

\section{DFT OF WINDOWED SIGNAL}

Consider the DFT of a windowed version of $x(k)$. In this paper, we assume that an arbitrary cosine-sum window function is employed, which has the form

$$
w(k)=\alpha_{0}+\sum_{p=1}^{P} \alpha_{p} e^{j 2 \pi \frac{p}{N} k}+\sum_{p=1}^{P} \alpha_{p} e^{-j 2 \pi \frac{p}{N} k},
$$

where $P$ is the number of cosine components of $w(k)$, and $\alpha_{p}(p=$ $0, \ldots, P)$ expresses a weight of the $p$ th cosine component. Examples of $P$ and $\alpha_{p}$ are shown in Table 1. The cosine-sum window functions can include the case of without windowing as the rectangular window. Using Eq. (7), the DFT of $x(k) w(k)$ can be represented by

$$
X_{w}(m)=\alpha_{0} X(m)+\sum_{p=1}^{P} \alpha_{p} X(m+p)+\sum_{p=1}^{P} \alpha_{p} X(m-p) .
$$

Hence, the windowed version of Eq. (6) is formulated by

$$
\begin{aligned}
X_{w}\left(m_{0}+l\right) & \\
=\quad & \alpha_{0} X\left(m_{0}+l\right)+\sum_{p=1}^{P} \alpha_{p} X\left(m_{0}+l+p\right) \\
& +\sum_{p=1}^{P} \alpha_{p} X\left(m_{0}+l-p\right) \\
=\quad & \tilde{a}\left\{\frac{\alpha_{0}\left(c_{l}+j s_{l}\right)}{c_{l} \tilde{s}-s_{l} \tilde{c}}+\sum_{p=1}^{P} \frac{\alpha_{p}\left(c_{p+l}+j s_{p+l}\right)}{c_{p+l} \tilde{s}-s_{p+l} \tilde{c}}\right. \\
& \left.+\sum_{p=1}^{P} \frac{\alpha_{p}\left(c_{p-l}-j s_{p-l}\right)}{c_{p-l} \tilde{s}+s_{p-l} \tilde{c}}\right\} .
\end{aligned}
$$

\section{PROPOSED APPROACH}

In this section, the Jacobsen estimator [4] which is for signals without windowing is extended to the windowed signals using the formulation of the DFT given by Eq. (9).
Table 1. Examples of weights for cosine-sum window functions.

\begin{tabular}{|c|c|c|c|c|}
\hline Window function & $P$ & $\alpha_{0}$ & $\alpha_{1}$ & $\alpha_{2}$ \\
\hline \hline Rectangular & 0 & 1 & - & - \\
Hanning & 1 & 0.5 & -0.25 & - \\
Hamming & 1 & 0.54 & -0.23 & - \\
Blackman & 2 & 0.42 & -0.25 & 0.04 \\
\hline
\end{tabular}

\subsection{Original Jacobsen estimator}

The Jacobsen estimator [4] utilizes a function that is constructed from three DFT coefficients $Y\left(m_{0}-1\right), Y\left(m_{0}\right)$ and $Y\left(m_{0}+1\right)$ as

$$
\begin{aligned}
& g(Y)=\frac{g_{\text {num }}(Y)}{g_{\text {den }}(Y)} \\
& \left(\begin{array}{l}
g_{\text {num }}(Y)=Y\left(m_{0}-1\right)-Y\left(m_{0}+1\right) \\
g_{\text {den }}(Y)=2 Y\left(m_{0}\right)-Y\left(m_{0}-1\right)-Y\left(m_{0}+1\right)
\end{array}\right)
\end{aligned}
$$

where $Y(m)$ is the DFT of $y(k)$. Comparing $g(Y)$ with the noiseless case, i.e., $g(X), \delta$ can be estimated. The estimator is given by solving the following equation:

$$
\operatorname{Re}\{g(Y)\}=\operatorname{Re}\{g(X)\}
$$

where $\operatorname{Re}\{\cdot\}$ means the real part of a complex number. As described later, several solutions to Eq. (11) have been developed so far [4-6].

Instead of considering $Y(m)$ and $X(m)$, we adopt the windowed signals for Eq. (11) as

$$
\operatorname{Re}\left\{g\left(Y_{w}\right)\right\}=\operatorname{Re}\left\{g\left(X_{w}\right)\right\}
$$

where $Y_{w}(m)$ is the DFT of $y(k) w(k)$. An analytical solution to Eq. (12) is derived in the following subsections.

\subsection{Extended Jacobsen estimator for $P=0$ case}

When $P=0$, it is identical to using signals without windowing. In other words, $w(k)$ is the rectangular window and the numerator and denominator polynomials of $g\left(X_{w}\right)$ in Eq. (12) are

$$
g_{n u m}\left(X_{w}\right)=\frac{-2 \tilde{a} \alpha_{0} s_{1} c_{1}(\tilde{c}+j \tilde{s})}{\tilde{c}^{2}\left(c_{1}^{2} \tilde{t}^{2}-s_{1}^{2}\right)}
$$

and

$$
g_{d e n}\left(X_{w}\right)=\frac{-2 \tilde{a} \alpha_{0} s_{1}^{2}(\tilde{c}+j \tilde{s})}{\tilde{c}^{2} \tilde{t}\left(c_{1}^{2} \tilde{t}^{2}-s_{1}^{2}\right)},
$$

respectively, where $\tilde{t}=\tan \left(\pi \frac{\delta}{N}\right)$. In the above simplification, we utilize

$$
\frac{c_{l}-j s_{l}}{c_{l} \tilde{s}+s_{l} \tilde{c}}-\frac{c_{l}+j s_{l}}{c_{l} \tilde{s}-s_{l} \tilde{c}}=-\frac{2 s_{l} c_{l}(\tilde{c}+j \tilde{s})}{\tilde{c}^{2}\left(c_{l}^{2} \tilde{t}^{2}-s_{l}^{2}\right)},
$$

and

$$
\frac{2}{\tilde{s}}-\frac{c_{l}-j s_{l}}{c_{l} \tilde{s}+s_{l} \tilde{c}}-\frac{c_{l}+j s_{l}}{c_{l} \tilde{s}-s_{l} \tilde{c}}=-\frac{2 s_{l}^{2}(\tilde{c}+j \tilde{s})}{\tilde{c}^{2} \tilde{t}\left(c_{l}^{2} \tilde{t}^{2}-s_{l}^{2}\right)} .
$$

Substituting Eqs. (13) and (14) into Eq. (10), we have a real-valued function

$$
g\left(X_{w}\right)=\frac{\tilde{t}}{t_{1}}
$$

where $t_{1}=\tan \left(\frac{\pi}{N}\right)$. Finally, substituting Eq. (17) into Eq. (12), an estimate of $\delta$ is given by

$$
\hat{\delta}=\frac{N}{\pi} \tan ^{-1}\left(t_{1} \operatorname{Re}\left\{g\left(Y_{w}\right)\right\}\right)
$$

Eq. (18) is identical to the analytical solution to the original Jacobsen estimator in [6]. 


\subsection{Extended Jacobsen estimator for $P=1$ case}

Hanning and Hamming windows which are commonly used window functions are classified into $P=1$ case. In this case, using Eqs. (9), (15) and (16), $g_{\text {num }}\left(X_{w}\right)$ and $g_{d e n}\left(X_{w}\right)$ can be formulated by

$$
g_{\text {num }}\left(X_{w}\right)=\frac{-2 \tilde{a}(\tilde{c}+j \tilde{s})}{\tilde{c}^{2}}\left\{\frac{\alpha_{0} s_{1} c_{1}}{c_{1}^{2} \tilde{t}^{2}-s_{1}^{2}}+\frac{\alpha_{1} s_{2} c_{2}}{c_{2}^{2} \tilde{t}^{2}-s_{2}^{2}}\right\},
$$

and

$$
g_{d e n}\left(X_{w}\right)=\frac{-2 \tilde{a}(\tilde{c}+j \tilde{s})}{\tilde{c}^{2} \tilde{t}}\left\{\frac{\left(\alpha_{0}-2 \alpha_{1}\right) s_{1}^{2}}{c_{1}^{2} \tilde{t}^{2}-s_{1}^{2}}+\frac{\alpha_{1} s_{2}^{2}}{c_{2}^{2} \tilde{t}^{2}-s_{2}^{2}}\right\},
$$

respectively. Because $s_{1}^{2}=\frac{1}{2}\left(1-c_{2}\right), c_{1}^{2}=\frac{1}{2}\left(1+c_{2}\right)$, and $s_{2}=2 s_{1} c_{1}, g\left(X_{w}\right)=\frac{g_{n u m}\left(X_{w}\right)}{g_{\text {den }}\left(X_{w}\right)}$ becomes a real-valued function represented as

$$
\begin{aligned}
& g\left(X_{w}\right)=\frac{\gamma_{3} \tilde{t}^{3}+\gamma_{1} \tilde{t}}{t_{1}\left(\gamma_{2} \tilde{t}^{2}+\gamma_{0}\right)} \\
& \left(\begin{array}{l}
\gamma_{3}=\left(\alpha_{0}+\alpha_{1}\right) c_{2}^{2}+\alpha_{1} c_{2} \\
\gamma_{2}=\left(\alpha_{0}-\alpha_{1}\right) c_{2}^{2}+2 \alpha_{1} c_{2}+\alpha_{1} \\
\gamma_{1}=\left(\alpha_{0}+\alpha_{1}\right) c_{2}^{2}-\alpha_{1} c_{2}-\alpha_{0} \\
\gamma_{0}=\left(\alpha_{0}-\alpha_{1}\right) c_{2}^{2}-\left(\alpha_{0}-\alpha_{1}\right)
\end{array}\right) .
\end{aligned}
$$

Substituting Eq. (21) into Eq. (12), we obtain a cubic equation

$$
\gamma_{3} \tilde{t}^{3}-\operatorname{Re}\left\{g\left(Y_{w}\right)\right\} t_{1} \gamma_{2} \tilde{t}^{2}+\gamma_{1} \tilde{t}-\operatorname{Re}\left\{g\left(Y_{w}\right)\right\} t_{1} \gamma_{0}=0 .
$$

When the Hanning window is used, $\alpha_{1}=-\frac{1}{2} \alpha_{0}$ and therefore Eq. (22) is further simplified to

$$
\begin{aligned}
& c_{2} \tilde{t}^{3}- \operatorname{Re}\left\{g\left(Y_{w}\right)\right\} t_{1}\left(3 c_{2}+1\right) \tilde{t}^{2} \\
&+\left(c_{2}+2\right) \tilde{t}-\operatorname{Re}\left\{g\left(Y_{w}\right)\right\} t_{1}\left(3 c_{2}+3\right)=0 .
\end{aligned}
$$

Assuming that $\operatorname{Re}\left\{g\left(Y_{w}\right)\right\} \simeq \frac{\delta}{2}$, which is obtained from the result in [9], and $N \geq 4$, it is clear that $0 \leq \operatorname{Re}\left\{g\left(Y_{w}\right)\right\}^{2} t_{1}^{2} \leq \frac{1}{16}$. Then, Eq. (23) is negative when

$$
c_{2}>\frac{-3+3 \operatorname{Re}\left\{g\left(Y_{w}\right)\right\}^{2} t_{1}^{2}+\sqrt{9-15 \operatorname{Re}\left\{g\left(Y_{w}\right)\right\}^{2} t_{1}^{2}}}{3-9 \operatorname{Re}\left\{g\left(Y_{w}\right)\right\}^{2} t_{1}^{2}} .
$$

From Eq. (24), Eq. (23) has one real root when $N>4.0284$. Hence, an estimate of $\delta$ for the Hanning window is given by

$$
\hat{\delta}=\frac{N}{\pi} \tan ^{-1}(r)
$$

where $r$ is a real root of Eq. (23), which can be obtained analytically [15].

Other window functions for $P=1$ give at least one real root of Eq. (22). In this case, the number of real roots can be investigated using a discriminant and all real roots have closed forms [15]. Note that, when Eq. (22) has three real roots, the second root in [15] is adopted because other real roots are out of the range of $\delta$. Acquiring a real root $r$ of Eq. (22), an estimate of $\delta$ can be obtained by Eq. (25).

\subsection{Extended Jacobsen estimator for $P \geq 2$ cases}

Generalizing the result of the $P=1$ case, $g_{\text {num }}\left(X_{w}\right)$ and $g_{\text {den }}\left(X_{w}\right)$ can be extended to the $P \geq 2$ cases. For $P \geq 2, g_{\text {num }}\left(X_{w}\right)$ and $g_{\text {den }}\left(X_{w}\right)$ are expressed by

$$
g_{n u m}\left(X_{w}\right)=\frac{-2 \tilde{a}(\tilde{c}+j \tilde{s})}{\tilde{c}^{2}} \tilde{g}_{n u m}
$$

and

$$
g_{d e n}\left(X_{w}\right)=\frac{-2 \tilde{a}(\tilde{c}+j \tilde{s})}{\tilde{c}^{2} \tilde{t}} \tilde{g}_{d e n}
$$

respectively, where $\tilde{g}_{n u m}$ and $\tilde{g}_{d e n}$ are defined by

$$
\tilde{g}_{\text {num }}=\frac{\alpha_{0} s_{1} c_{1}}{c_{1}^{2} \tilde{t}^{2}-s_{1}^{2}}+\sum_{p=1}^{P} \frac{\alpha_{p} s_{p+1} c_{p+1}}{c_{p+1}^{2} \tilde{t}^{2}-s_{p+1}^{2}}-\sum_{p=2}^{P} \frac{\alpha_{p} s_{p-1} c_{p-1}}{c_{p-1}^{2} \tilde{t}^{2}-s_{p-1}^{2}},
$$

and

$$
\begin{aligned}
\tilde{g}_{d e n}= & \frac{\alpha_{0} s_{1}^{2}}{c_{1}^{2} \tilde{t}^{2}-s_{1}^{2}}-2 \sum_{p=1}^{P} \frac{\alpha_{p} s_{p}^{2}}{c_{p}^{2} \tilde{t}^{2}-s_{p}^{2}} \\
& +\sum_{p=1}^{P} \frac{\alpha_{p} s_{p+1}^{2}}{c_{p+1}^{2} \tilde{t}^{2}-s_{p+1}^{2}}+\sum_{p=2}^{P} \frac{\alpha_{p} s_{p-1}^{2}}{c_{p-1}^{2} \tilde{t}^{2}-s_{p-1}^{2}}
\end{aligned}
$$

respectively. Therefore, $g\left(X_{w}\right)$ with $P \geq 2$ yields a real-valued function

$$
g\left(X_{w}\right)=\frac{\tilde{g}_{\text {num }} \tilde{t}}{\tilde{g}_{\text {den }}}
$$

Substituting Eq. (30) into Eq. (12), we have an algebraic equation of degree $(2 P+1)$ as follows:

$$
\tilde{g}_{\text {num }} \tilde{t} \prod_{p=1}^{P}\left(c_{p}^{2} \tilde{t}^{2}-s_{p}^{2}\right)-\operatorname{Re}\left\{g\left(Y_{w}\right)\right\} \tilde{g}_{d e n} \prod_{p=1}^{P}\left(c_{p}^{2} \tilde{t}^{2}-s_{p}^{2}\right)=0 .
$$

Clearly, Eq. (31) has at least one real root because its coefficients are all real numbers and the degree is an odd number. In general, Eq. (31) with $P \geq 2$ isn't analytically solvable. However, they can be obtained similarly to analytical solutions using root-finding algorithms. If multiple real roots are calculated, we extract a root that has the smallest absolute value in all real roots. Once $r$ is obtained, an estimate of $\delta$ is finally given by Eq. (25).

\subsection{Related works}

The original Jacobsen estimator [4] was developed for signals without windowing. In this case, an estimate of $\delta$ is given by solving Eq. (11). The solution to Eq. (11) was firstly developed with some approximations of the trigonometric functions under the assumption that $\frac{\pi}{N} \ll 1$ as

$$
\hat{\delta}_{j a c}=\operatorname{Re}\{g(Y)\} .
$$

Although this estimator has a noticeable advantage with respect to computational efficiency, a relatively large estimation error occurs due to the estimator bias. After some improved estimators were proposed (e.g., [5]), an analytical solution to Eq. (11) with no approximation was found in [6] as

$$
\hat{\delta}_{\text {ana }}=\frac{N}{\pi} \tan ^{-1}\left(t_{1} \operatorname{Re}\{g(Y)\}\right)
$$

As aforementioned, our estimator when $P=0$ is identical to this analytical solution. However, these estimators are applied only to the signals without windowing.

For the windowed signals, the Candan estimator that exploits the Taylor series expansion was developed [9]. In this estimator, $X_{w}(m)$ is reformulated using the first degree Taylor polynomial of the DFT of $w(k)$. This approximated reformulation leads to a simple estimator

$$
\hat{\delta}_{c a n}=\beta \operatorname{Re}\left\{g\left(Y_{w}\right)\right\},
$$


where $\beta$ is a constant depending on the DFT of $w(k)$ and its first derivative. For example, $\beta \simeq 2$ for the Hanning window [9]. We utilize this result for the derivation of Eq. (24). The Candan estimator can be applied to an arbitrary window function. However, the estimation error caused by the estimator bias becomes significant when the SNR is high. An alternative approach to the windowed version of the Jacobsen estimator is the Liao estimator [10]. In the Liao estimator, considering the Hanning window and altering $g\left(Y_{w}\right)$ as

$$
g_{\text {liao }}\left(Y_{w}\right)=\frac{Y_{w}\left(m_{0}-1\right)-Y_{w}\left(m_{0}+1\right)}{\zeta Y_{w}\left(m_{0}\right)-\eta Y_{w}\left(m_{0}-1\right)-\eta Y_{w}\left(m_{0}+1\right)},
$$

where $\zeta=2 \cot \left(\frac{\pi}{N}\right)-2 \cot \left(\frac{2 \pi}{N}\right)$ and $\eta=\cot \left(\frac{2 \pi}{N}\right)$, the following analytical solution was achieved:

$$
\hat{\delta}_{\text {liao }}=\frac{N}{\pi} \tan ^{-1}\left(\operatorname{Re}\left\{g_{\text {liao }}\left(Y_{w}\right)\right\}\right) .
$$

Because Eq. (36) is derived with no approximation, the performance is improved when the SNR is high. However, the Liao estimator can be applied only to the Hanning-windowed signals.

\section{SIMULATION RESULTS}

In order to validate the proposed estimator, two simulations were carried out. First, we used a single complex sinusoid buried in additive complex white Gaussian noise as $y(k)$. In this simulation, the Hanning window was employed in order to compare the performance of the proposed method with the Liao estimator [10] and the Candan estimator [9]. Also the Cramér-Rao lower bound (CRLB) for estimation of $f$ [16] and the analytical solution to the Jacobsen estimator without windowing [6] were compared. Second, an observed signal composed of two complex sinusoids and complex white Gaussian noise was used in order to investigate the effect of a window function. The lower frequency of the complex sinusoids was the target frequency. In this simulation, our estimator, the Candan estimator [9], and the analytical solution to the Jacobsen estimator without windowing was compared. For the proposed estimator and the Candan estimator, the Hamming window or the Blackman window was adopted. In these simulations, based on [9], parameters were set to $N=16, \delta=0.45, m_{0}=5, a=1$, and a random number for $b$. For the multiple complex sinusoids, the amplitude, the initial phase, and the frequency of the higher-frequency sinusoid were $a \sqrt{10}$, a random number, and $\frac{1}{N}\left(m_{0}+\delta+4.5\right)$, respectively, based on [9]. In the Candan estimator, the iteration procedure wasn't employed because the estimator bias was assessed. 100 trials using i.i.d. noise were carried out and estimated frequencies were evaluated by the root-mean-square error (RMSE). Because our method focuses on the reduction of the estimator bias, the relatively high SNRs were investigated.

Fig. 1 depicts the simulation results of the single complex sinusoid case. In this figure, Jacobsen (Analytical) gave the slightly lower RMSEs than the others because windowing yields the SNR loss in each DFT coefficient in $g\left(Y_{w}\right)[9,17]$. Except for Jacobsen (Analytical), it is clearly seen that the RMSEs by Proposed (Hanning) were almost the same as Lian (Hanning) and they were better than Candan (Hanning) when the SNR is high. These results indicate that the proposed estimator is the analytical solution to the Jacobsen estimator with a window function for the single complex sinusoid case. The results of the multiple complex sinusoids case is illustrated in Fig. 2. From this figure, it can be observed that a window function improves the RMSE for the multiple complex sinusoids case. Especially when the SNR is relatively high, our esti-

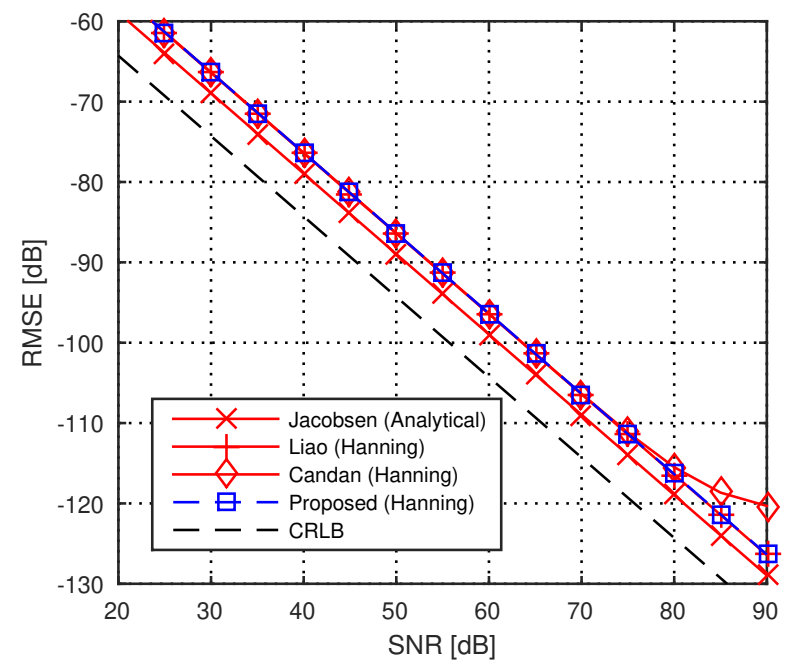

Fig. 1. Performance comparison in terms of RMSEs for the single complex sinusoid case.

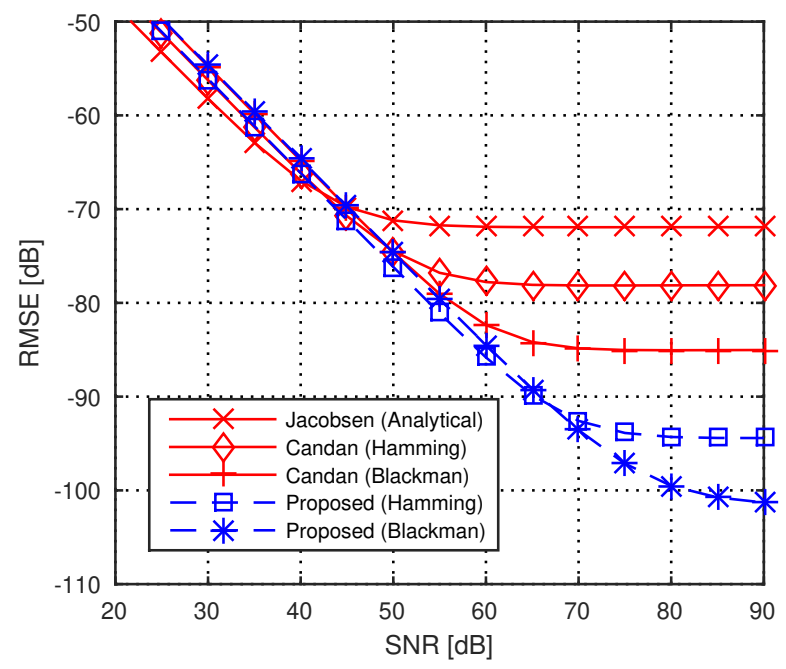

Fig. 2. Performance comparison in terms of RMSEs for the multiple complex sinusoids case.

mator is significantly better than the Candan estimator by virtue of elimination of the estimator bias.

\section{CONCLUSIONS}

We have extended the Jacobsen estimator to the windowed signals and it has been revealed that the estimator results in an algebraic equation with no approximation. Simulation results have shown that the proposed estimator improves the performance by removing the estimator bias when the SNR is high, and it is effective even in the frequency estimation of the multiple complex sinusoids. Future works include extension of other estimators based on the interpolated DFT to the windowed signals and further extension to window functions except for the cosine-sum windows. 


\section{REFERENCES}

[1] B. G. Quinn, "Estimating frequency by interpolation using Fourier coefficients," IEEE Trans. Signal Processing, vol. 42, no. 5, pp. 1264-1268, May 1994.

[2] M. D. Macleod, "Fast nearly ML estimation of the parameters of real or complex single tones or resolved multiple tones," IEEE Trans. Signal Processing, vol. 46, no. 1, pp. 141-148, January 1998.

[3] E. Aboutanios and B. Mulgrew, "Iterative frequency estimation by interpolation of Fourier coefficients," IEEE Trans. Signal Processing, vol. 53, no. 4, pp. 1237-1242, April 2005.

[4] E. Jacobsen and P. Kootsookos, "Fast, accurate frequency estimators," IEEE Signal Processing Magazine, vol. 24, no. 3, pp. 123-125, May 2007.

[5] C. Candan, "A method for fine resolution frequency estimation from three DFT samples," IEEE Signal Processing Letters, vol. 18, no. 6, pp. 351-354, June 2011.

[6] J.-R. Liao and S. Lo, "Analytical solutions for frequency estimators by interpolation of DFT coefficients," Signal Processing, vol. 100, pp. 93-100, July 2014.

[7] X. Liang, A. Liu, X. Pan, Q. Zhang, and F. Chen, "A new and accurate estimator with analytical expression for frequency estimation," IEEE Communications Letters, vol. 20, no. 1, pp. 105-108, January 2016.

[8] A. Serbes, "Fast and efficient sinusoidal frequency estimation by using the DFT coefficients," IEEE Trans. Communications, vol. 67, no. 3, pp. 2333-2337, March 2019.

[9] C. Candan, "Fine resolution frequency estimation from three DFT samples: Case of windowed data," Signal Processing, vol. 114, pp. 245-250, September 2015.

[10] J.-R. Liao, "Analytical solution of DFT interpolated frequency estimator for Hanning windowed signal," in Proc. 2015 15th International Symposium on Communications and Information Technologies (ISCIT), Nara, Japan, October 2015, pp. 177180.

[11] J. Luo, Z. Xie, and M. Xie, "Interpolated DFT algorithms with zero padding for classic windows," Mechanical Systems and Signal Processing, vol. 70-71, pp. 1011-1025, March 2016.

[12] C. Candan, "Analysis and further improvement of fine resolution frequency estimation method from three DFT samples," IEEE Signal Processing Letters, vol. 20, no. 9, pp. 913-916, September 2013.

[13] M. D. Macleod, "Fast high accuracy estimation of multiple cisoids in noise," Signal Processing V: Theories and Applications, pp. 333-336, September 1990.

[14] D. C. Rife and G. A. Vincent, "Use of the discrete Fourier transform in the measurement of frequencies and levels of tones," The Bell System Technical Journal, vol. 49, no. 2, pp. 197-228, February 1970.

[15] R. W. D. Nickalls, "A new approach to solving the cubic: Cardan's solution revealed," The Mathematical Gazette, vol. 77, no. 480, pp. 354-359, November 1993.

[16] S. M. Kay, Fundamentals of Statistical Signal Processing, Volume 1: Estimation Theory, Prentice Hall, New Jersey, 1993.

[17] B. Porat, A Course in Digital Signal Processing, John Wiley \& Sons, New York, 1996. 